\title{
THE USE OF FRACTIONAL INTEGRATION AND \\ DIFFERENTIATION FOR OBTAINING CERTAIN EXPANSIONS IN TERMS OF BESSEL FUNCTIONS OR OF SINES AND COSINES
}

BY W. O. PENNELL

1. Introduction. Under certain conditions fractional integration or differentiation of a sine function will lead to a Bessel function and vice versa. Likewise the fractional integration or differentiation of a cosine function will lead to Struve's function. It follows, when the process is legitimate, that a known expansion in sines can be converted to an expansion in Bessel functions, or a known expansion in Bessel functions can be converted to a sine expansion, by the simple expedient of term-by-term fractional integration or differentiation.

2. Fractional Integration and Differentiation of Sine and Cosine. Fractional integration* with the Heaviside operator $p$ is given by the equation

$$
p^{-\nu} f(x)=\int_{0}^{x} \frac{(x-\lambda)^{\nu-1}}{\Gamma(\nu)} f(\lambda) d \lambda,
$$

where $\nu>0$. Fractional differentiation* is given by

$$
p^{\nu} f(x)=\frac{d^{b}}{d x^{b}} \int_{0}^{x} \frac{(x-\lambda)^{c-1}}{\Gamma(c)} f(\lambda) d \lambda,
$$

where $\nu>0,0<c<1, b$ is a positive integer, and $\nu=b-c$. If (1) is applied to $f(x)=x^{n}$ the result is

$$
p^{-\nu} x^{n}=\frac{\Gamma(n+1) x^{n+\nu}}{\Gamma(n+\nu+1)}, \quad(\nu>0, n>-1) .
$$

If (2) is applied to $x^{n}$,

$$
p^{\nu} x^{n}=\frac{\Gamma(n+1) x^{n-\nu}}{\Gamma(n-\nu+1)}, \quad(\nu>0, n>-1) .
$$

* For bibliography on fractional integration see H. T. Davis, The application of fractional operators to functional equations, American Journal of Mathematics, vol. 49 (1927), pp. 123-142. 
If $n-\nu+1$ is zero or a negative integer, the right hand side of (4) is to be interpreted as the limit which that expression approaches as $\nu$ approaches the value which causes $n-\nu+1$ to become zero or a negative in teger.

It follows from (3) and (4) that $p^{\nu} x^{n}=\Gamma(n+1) x^{n-\nu} / \Gamma(n-\nu+1)$ for all values* of $\nu$, for $n>-1$.

By applying process (3) or (4) term by term to the power series representing the sine or cosine we get

$$
\begin{aligned}
& \text { (5) } p^{-\nu+1 / 2} \sin a x^{1 / 2}=\left(\frac{2}{a}\right)^{\nu-1} \pi^{1 / 2} x^{\nu / 2} J_{\nu}\left(a x^{1 / 2}\right), \\
& \text { (6) } p^{-\nu+1 / 2} \cos a x^{1 / 2}=\frac{x^{\nu-1 / 2}}{\Gamma\left(\nu+\frac{1}{2}\right)}-\left(\frac{2}{a}\right)^{\nu-1} x^{\nu / 2} \pi^{1 / 2} H_{\nu}\left(a x^{1 / 2}\right),
\end{aligned}
$$

where $H_{\nu}$ denotes Struve's function of order $\nu$. That the term-byterm fractional integration and differentiation is legitimate may be shown as follows. Since the power series for $\sin a x^{1 / 2}$ is uniformly convergent for all finite values of $x$ it may be written as

$$
\sin a x^{1 / 2}=\sum_{n=1,2}^{n}(-1)^{n-1} \frac{\left(a x^{1 / 2}\right)^{2 n-1}}{(2 n-1) !}+R_{n},
$$

where $\left|R_{n}\right|<\epsilon, \epsilon$ being chosen arbitrarily small and positive, and corresponding to it is a positive integer $n$, independent of $x$. By applying process (1), fractional integration, to both sides of (7), we obtain

$$
p^{-\nu+1 / 2} \sin a x^{1 / 2}=\sum_{n=1,2}^{n}(-1)^{n-1} p^{-\nu+1 / 2} \frac{\left(a x^{1 / 2}\right)^{2 n-1}}{(2 n-1) !}+p^{-\nu+1 / 2} R_{n} .
$$

But

$$
\left|p^{-\nu+1 / 2} R_{n}\right|<\left|\frac{\epsilon x^{\nu-1 / 2}}{\Gamma\left(\nu+\frac{1}{2}\right)}\right|,
$$

and, since $\nu \geqq+1 / 2$, this term can be made as small as we like, for any finite value of $x$.

Fractional differentiation, shown by (2), is fractional integration followed by ordinary differentiation. Since the fractional integral of $\sin a x^{1 / 2}$ or $\cos a x^{1 / 2}$ leads to a Bessel function of the first kind or Struve's function, and since the power series repre-

* Including $\nu=0$, as $p^{0}$. will be defined as the identical operation. 
senting these functions may be differentiated term by term, it follows that the process leading to (5) and (6) is legitimate for all real values of $\nu$, positive or negative.

By transposing the operator in (5) and (6) we get

$$
\begin{aligned}
& p^{\nu-1 / 2}\left(\frac{2}{a}\right)^{\nu-1} \pi^{1 / 2} x^{\nu / 2} J_{\nu}\left(a x^{1 / 2}\right)=\sin a x^{1 / 2}, \\
& p^{\nu-1 / 2}\left(\frac{2}{a}\right)^{\nu-1} \pi^{1 / 2} x^{\nu / 2} H_{\nu}\left(a x^{1 / 2}\right)=1-\cos a x^{1 / 2} .
\end{aligned}
$$

3. Fractional Integration of Fourier-Bessel Series. This series for $f(x)$ is given by

$$
f(x)=\sum_{m=1,2}^{\infty} a_{m} J_{\nu}\left(j_{m} x\right), \quad\left(0<x<1, \nu \geqq-\frac{1}{2}\right),
$$

where

$$
a_{m}=\frac{2}{J_{\nu+1}^{2}\left(j_{m}\right)} \int_{0}^{1} \lambda f(\lambda) J_{\nu}\left(j_{m} \lambda\right) d \lambda,
$$

and where $j_{m}$ is the $m$ th positive root of $J_{\nu}(x)=0$. From (10),

$$
x^{\nu / 2} f\left(x^{1 / 2}\right)=\sum_{m=1,2}^{\infty} a_{m} x^{\nu / 2} J_{\nu}\left(j_{m} x^{1 / 2}\right) .
$$

It can be shown* that (12), with certain restrictions on $f(x)$, is uniformly convergent in the interval $0<x<1$, for $\nu \geqq 0$. The series may, therefore, be fractionally integrated term by term. We get by applying process (8) to (12)

$$
p^{\nu-1 / 2} x^{\nu / 2} f\left(x^{1 / 2}\right)=\frac{1}{\pi^{1 / 2}} \sum_{m=1,2}^{\infty} a_{m}\left(\frac{2}{j_{m}}\right)^{1-\nu} \sin \left(j_{m} x^{1 / 2}\right), \quad\left(\frac{1}{2} \geqq \nu \geqq 0\right) .
$$

If we put

then

$$
\phi\left(x^{1 / 2}\right)=p^{\nu-1 / 2} x^{\nu / 2} f\left(x^{1 / 2}\right),
$$

$$
f\left(x^{1 / 2}\right)=x^{-\nu / 2} p^{-\nu+1 / 2} \phi\left(x^{1 / 2}\right),
$$

and

$$
\begin{aligned}
\phi(x)=\frac{1}{\pi^{1 / 2}} \sum_{m=1,2}^{\infty} a_{m}\left(\frac{2}{j_{m}}\right)^{1-\nu} \sin \left(j_{m} x\right), \\
\left(\frac{1}{2} \geqq \nu \geqq 0,0<x<1\right),
\end{aligned}
$$

* See Watson, Theory of Bessel Functions, pp. 594-615. Also C. N. Moore, On the uniform convergence of the developments in Bessel functions, Transactions of this Society, vol. 12 (1911), pp. 181-206. 
where $a_{m}$ is given by (11). The function $f(x)$ is subject to the restrictions sufficient for a function to be expansible by a FourierBessel series. The function $\phi(x)$ is given by (13). If $f(x)=\sum a_{n} x^{n}$, a power series, (13) shows that

$$
\psi(x)=\sum_{n} a_{n} \frac{\Gamma(n / 2+\nu / 2+1)}{\Gamma(n / 2-\nu / 2+3 / 2)} x^{n-\nu+1} .
$$

We have here the expansion of a function in a series of sine terms, and the expansion is not periodic. As far as the author is aware the expansion (14) has never before been published.

ExAmple. Let us start with the known expansion

$$
x^{\nu}=\sum_{m=1,2}^{\infty} \ldots \frac{2 J_{\nu}\left(j_{m} x\right)}{j_{m} J_{\nu+1}\left(j_{m}\right)}, \quad\left(\nu \geqq-\frac{1}{2}, 0 \leqq x<1\right) .
$$

Substituting $x^{1 / 2}$ for $x$, multiplying both sides by $x^{\nu / 2}$, operating on both sides by $p^{\nu-1 / 2}$, and finally replacing $x^{1 / 2}$ by $x$, we may write (15) in the form

$$
x=\sum_{m=1,2}^{\infty} \frac{1}{2^{\nu-1}\left(j_{m}\right)^{2-\nu} J_{\nu+1}\left(j_{m}\right) \Gamma(\nu+1)} \sin j_{m} x,
$$

where $0 \leqq x<1,0 \leqq \nu \leqq 1 / 2$, and $j_{m}$ is the $m$ th positive root of $J_{\nu}(x)=0$.

4. Fractional Integration of Fourier Series. Let us consider the Fourier series

$$
f(x)=\frac{1}{2} b_{0}+\sum_{n=1,2}^{\infty}\left[a_{n} \sin \frac{n \pi x}{c}+b_{n} \cos \frac{n \pi x}{c}\right]
$$

where

$$
a_{n}=\frac{1}{c} \int_{-c}^{c} f(x) \sin \frac{n \pi x}{c} d x, b_{n}=\frac{1}{c} \int_{-c}^{c} f(x) \cos \frac{n \pi x}{c} d x .
$$

In (17), replacing $x$ by $x^{1 / 2}$ and operating on both sides by $p^{-\nu+1 / 2}$, we find

$$
\begin{array}{r}
p^{-\nu+1 / 2} f\left(x^{1 / 2}\right)=\frac{1}{2} b_{0} \frac{x^{\nu-1 / 2}}{\Gamma\left(\nu+\frac{1}{2}\right)}+\sum_{n=1,2}^{\infty} \pi^{1 / 2}\left(\frac{2 c}{n \pi}\right)^{\nu-1} x^{\nu / 2} \\
\cdot\left[a_{n} J_{\nu}\left(\frac{n \pi x^{1 / 2}}{c}\right)-b_{n} H_{\nu}\left(\frac{n \pi x^{1 / 2}}{c}\right)\right]+\sum_{n=1,2}^{\infty} b_{n} \frac{x^{\nu-1 / 2}}{\Gamma\left(\nu+\frac{1}{2}\right)}, \\
x^{-\nu / 2} p^{-\nu+1 / 2} f\left(x^{1 / 2}\right)=\frac{f(0) x^{\nu / 2-1 / 2}}{\Gamma\left(\nu+\frac{1}{2}\right)}+\pi^{1 / 2}\left(\frac{2 c}{\pi}\right)^{\nu-1} \sum_{n=1,2}^{\infty} \frac{1}{n^{\nu-1}} \\
\cdot\left[a_{n} J_{\nu}\left(\frac{n \pi x^{1 / 2}}{c}\right)-b_{n} H_{\nu}\left(\frac{n \pi x^{1 / 2}}{c}\right)\right],
\end{array}
$$


where $f(0)=\frac{1}{2}[f(+0)+f(-0)]$. Since the integral of a function defined and satisfying Dirichlet's conditions in a given interval is equal to the term-by-term integral of the corresponding Fourier series, it follows that the fractional integral of the function will equal the term-by-term fractional integral of the Fourier series. This is so because fractional integration of order $\nu-1 / 2$ (see equation (1)) is equivalent to multiplying each term by $(x-\lambda)^{\nu-3 / 2} / \Gamma(\nu-1 / 2)$ and then integrating the resulting series term by term. The above process which results in (18) is therefore legitimate when $\nu>1 / 2$, that is, for fractional integration.

When $\nu<1 / 2$ we have fractional differentiation. Suppose $-1 / 2<\nu<1 / 2$; then $p^{-\nu+1 / 2}$ may be written $p^{-\nu-1 / 2} p$, which represents first differentiation and then fractional integration. Hence when the Fourier series is of such a character that it can be differentiated term by term, then the process $p^{-\nu-1 / 2}$, which is fractional integration, can be applied to the resulting Fourier series. That is, the original Fourier series under these conditions can be fractionally differentiated and the result will equal the fractional derivative of the function represented by the series.

A known sufficient condition for term-by-term differentiation of a Fourier series in an interval $-a \leqq x \leqq a$ is that $f(x)$ shall have a derivative and shall be finite and continuous in the interval, and have only a finite number of maxima and minima, and that $f(a)=f(-a)$. Similar criteria will apply for $-3 / 2<\nu<-1 / 2$. Here $p^{-\nu+1 / 2}$ may be written $p^{-\nu-3 / 2} p^{2}$ and if the Fourier series may be differentiated twice, the fractional differentiation is legitimate and similar reasoning holds for lower values of $\nu$. Let

$$
x^{-\nu / 2} p^{-\nu+1 / 2} f\left(x^{1 / 2}\right)=\phi\left(x^{1 / 2}\right) .
$$

Then (18) becomes when $x$ is substituted for $x^{1 / 2}$

$$
\begin{aligned}
& \phi(x)=\frac{f(0) x^{\nu-1}}{\Gamma\left(\nu+\frac{1}{2}\right)} \\
& +\pi^{1 / 2}\left(\frac{2 c}{\pi}\right)^{\nu-1} \sum_{n=1,2}^{\infty} \frac{1}{n^{\nu-1}} \cdot\left[a_{n} J_{\nu}\left(\frac{n \pi x}{c}\right)-b_{n} H_{\nu}\left(\frac{n \pi x}{c}\right)\right],
\end{aligned}
$$

which is a generalized Schlömilch expansion of a function. For the validity of this expansion, the following conditions are sufficient. 
(1) The function $\phi(x)$ satisfies the condition that if $f\left(x^{1 / 2}\right)=p^{\nu-1 / 2} x^{\nu / 2} \phi\left(x^{1 / 2}\right)$, then $f(x)$ satisfies Dirichlet's conditions sufficient for representation of $f(x)$ by a Fourier series.

(2) $\nu \geqq 1 / 2$, provided $p^{-\nu+1 / 2}$ is taken as the identical operation when $\nu=1 / 2$.

(3) Or, if $1 / 2-n<\nu<1 / 2-(n-1),(n=1,2,3, \cdots)$, the function $f(x)$ satisfies the known conditions that $f^{n}(x)$ will equal the corresponding Fourier series when differentiated term by term $n$ times.

(4) The expansion is true for $-a<x<a$.

If $f(x)$ is represented by a Fourier sine series in the interval $0<x<c$, equation (19) becomes

$$
\phi(x)=\pi^{1 / 2}\left(\frac{2 c}{\pi}\right)^{\nu-1} \sum_{n=1,2}^{\infty} \frac{a_{n}}{n^{\nu-1}} J_{\nu}\left(\frac{n \pi x}{c}\right) .
$$

If $f(x)$ is represented by a Fourier cosine series in the interval $0<x<c$, equation (19) becomes

$$
\phi(x)=\frac{f(0) x^{\nu-1}}{\Gamma\left(\nu+\frac{1}{2}\right)}-\pi^{1 / 2}\left(\frac{2 c}{\pi}\right)^{\nu-1} \sum_{n=1,2}^{\infty} \frac{b_{n}}{n^{\nu-1}} H_{\nu}\left(\frac{n \pi x}{c}\right),
$$

where

$$
a_{n}=\frac{2}{c} \int_{0}^{c} f(x) \sin \frac{n \pi x}{c} d x, \quad b_{n}=\frac{2}{c} \int_{0}^{c} f(x) \cos \frac{n \pi x}{c} d x .
$$

Hence $\phi\left(x^{1 / 2}\right)=x^{-\nu / 2} p^{-\nu+1 / 2} f\left(x^{1 / 2}\right)$ and the same limitations hold as regards $f(x)$ and the values of $\nu$ apply as for (19).

Example. In (20), let us suppose that $f\left(x^{1 / 2}\right)=1$ if $0<x<c$, and $f\left(x^{1 / 2}\right)=-1$ if $c<x<2 c$. Then

$$
\phi\left(x^{1 / 2}\right)=x^{-\nu / 2} p^{-\nu+1 / 2}=\frac{x^{-\nu / 2} x^{\nu-1 / 2}}{\Gamma\left(\nu+\frac{1}{2}\right)}=\frac{x^{\nu / 2-1 / 2}}{\Gamma\left(\nu+\frac{1}{2}\right)},
$$

and $a_{n}=4 /(n \pi)$ for $n=1,3,5, \cdots$, and $a_{n}=0$ for $n=2,4, \cdots$. Hence

$$
\begin{aligned}
\phi(x)=\frac{x^{\nu-1}}{\Gamma\left(\nu+\frac{1}{2}\right)}=\pi^{1 / 2}\left(\frac{2 c}{\pi}\right)^{\nu-1} \sum_{n=1,3,5}^{\infty} \frac{4}{\pi n^{\nu}} J_{\nu}\left(\frac{n \pi x}{c}\right), \\
\left(0<x<c, \nu \geqq \frac{1}{2}\right) .
\end{aligned}
$$


Proceeding in exactly the same manner but starting with $f\left(x^{1 / 2}\right)=x^{1 / 2} / \Gamma(3 / 2)$, we obtain

$$
\begin{aligned}
& x^{\nu}=-\sum_{n=1,2}^{\infty}(-1)^{n}\left(\frac{2 c}{\pi}\right)^{\nu} \frac{2 \Gamma(\nu+1)}{n^{\nu}} J_{\nu}\left(\frac{n \pi x}{c}\right), \\
&(0<x<c, \nu \geqq 0) .
\end{aligned}
$$

5. Examples of Fourier Series with the Derived Schlömilch Series.

$$
\begin{aligned}
& \frac{\pi \sin a x}{2 \sin a \pi}= \sum_{n=1,2}^{\infty} \ldots \\
&(-\pi<x<\pi, a \text { not an integer }) . \\
& \frac{\pi a^{1-\nu}}{2 \sin a \pi} J_{\nu}(a x)=\sum_{n=1,2}^{\infty} \ldots \begin{array}{l}
n \sin n x \\
n^{2}-a^{2}
\end{array} \\
& \quad(-1)^{n-1} \frac{n J_{\nu}(n x)}{\left(n^{2}-a^{2}\right) n^{\nu-1}}, \\
&
\end{aligned}
$$

4) $\frac{\pi \cos a x}{2 \sin a \pi}=\frac{1}{2 a}+\sum_{n=1,2}^{\infty}(-1)^{n} \frac{a \cos n x}{a^{2}-n^{2}}$,

$$
(-\pi \leqq x \leqq \pi, a \text { not an integer). }
$$

$$
\begin{array}{r}
\frac{\pi H_{\nu}(a x)}{2 a^{\nu} \sin a \pi}=\sum_{n=1,2}^{\infty} \ldots \\
(-1)^{n} \frac{1}{\left(a^{2}-n^{2}\right) n^{\nu-1}} H_{\nu}(n x), \\
\left(-\pi \leqq x \leqq \pi, \nu \geqq-\frac{1}{2}\right) .
\end{array}
$$

(25) $\frac{\pi}{4} \sin x=\frac{1}{2}-\sum_{n=1,2}^{\infty} \frac{\cos 2 n x}{(2 n-1)(2 n+1)}, \quad(0 \leqq x \leqq \pi)$.

$$
\begin{array}{r}
J_{\nu}(x)=\frac{1}{2^{\nu-3} \pi} \sum_{n=1,2}^{\infty} \frac{1}{(2 n-1)(2 n+1) n^{\nu-1}} H_{\nu}(2 n x), \\
\left(0 \leqq x \leqq \pi, \nu \geqq-\frac{1}{2}\right) .
\end{array}
$$

$$
e^{i a x}=\frac{2}{\pi} \sum_{n=1,2 \ldots}^{\infty} \frac{n}{n^{2}-a^{2}}\left(1-\cos n \pi e^{i a \pi}\right) \sin n x,
$$

$(0<x<\pi, a$ not an integer $)$, 


$$
\begin{aligned}
& \frac{x^{\nu-1}}{\pi^{1 / 2} \Gamma\left(\nu+\frac{1}{2}\right)}-\left(\frac{2}{a}\right)^{\nu-1} H_{\nu}(a x)=-i\left(\frac{2}{a}\right)^{\nu-1} J_{\nu}(a x) \\
&+\frac{2}{\pi} \sum_{n=1,2}^{\infty} \ldots \frac{n}{n^{2}-a^{2}}\left(1-\cos n \pi e^{i a \pi}\right)\left(\frac{2}{n}\right)^{\nu-1} J_{\nu}(n x),
\end{aligned}
$$

where $0<x<\pi$ and $\nu \geqq 1 / 2$.

$$
\begin{gathered}
f(x)=a \text { if } 0<x<c / 2 ; f(x)=0 \text { if } x=c / 2 ; \\
f(x)=-a \text { if } c / 2<x<c . \\
\frac{4 a}{\pi} \sum_{n=1,2}^{\infty} \frac{1}{2 n-1} \sin \frac{2(2 n-1) \pi}{c} x=f(x),(0<x<c), \\
\frac{4 a}{\pi^{1 / 2}} \sum_{n=1,2}^{\infty} \frac{1}{(2 n-1)}\left[\frac{c}{(2 n-1) \pi}\right]^{\nu-1} J_{\nu}\left[\frac{2(2 n-1) \pi x}{c}\right] \\
=\frac{a x^{\nu-1}}{\Gamma\left(\nu+\frac{1}{2}\right)}, \quad(0<x \leqq c / 2), \\
=\frac{a x^{\nu-1}-2 a x^{-\nu}\left(x^{2}-c^{2} / 4\right)^{\nu-1 / 2}}{\Gamma\left(\nu+\frac{1}{2}\right)}, \quad\left(c / 2 \leqq x<c, \nu \geqq \frac{1}{2}\right) .
\end{gathered}
$$

It will be noted that the method described in this paper of obtaining the Schlömilch series from a corresponding Fourier series places no restriction on the upper value of $\nu$. In such literature on Schlömilch series as the author has seen, the upper limit of $\nu$ is $\nu<1 / 2$, that is, the order of the Bessel or Struve's function in the series $<1 / 2$.

St. Louis, MissourI 